\title{
Pulmonary graft-versus-host disease after autolo- gous hematopoietic stem cell transplantation
}

\author{
Sue In $\mathrm{Choi}^{1}$, Eun Joo Lee ${ }^{2}$, Dong Oh Kang ${ }^{1}$, Sang Yeub Lee ${ }^{2}$, Kwang Ho $\mathrm{In}^{2}$, Han-Kyeom Kim³,
} and Sanghoon Park ${ }^{4}$

\begin{abstract}
${ }^{1}$ Department of Internal Medicine, ${ }^{2}$ Division of Respiratory and Critical Care Medicine, Department of Internal Medicine, ${ }^{3}$ Department of Pathology, Korea University College of Medicine, Seoul; ${ }^{4}$ Department of Internal Medicine, KEPCO Medical Center, Seoul, Korea
\end{abstract}

Received: April 23, 2014

Revised : July 8, 2014

Accepted: August 5, 2014

\section{Correspondence to}

Eun Joo Lee, M.D.

Division of Respiratory and Critical Care Medicine, Department of Internal Medicine, Korea University Anam Hospital, 73 Inchon-ro, Seongbuk-gu, Seoul 02841, Korea Tel: +82-2-920-5048

Fax: +82-2-929-2045

E-mail:nanjung@korea.ac.kr
To the Editor,

Autologous graft-versus-host disease (auto-GVHD) is an autoimmune syndrome similar to acute GVHD that occurs after autologous hematopoietic stem cell transplantation (auto-HSCT). Clinically and histologically, it is indistinguishable from allogeneic GVHD (allo-GVHD), but it has a milder, self-limited course. Auto-GVHD was first described by Hood et al. [1] in 1987 and most commonly involves the skin and rarely the intestinal tract and liver. Here, we report the first case of pulmonary auto-GVHD in a Korean with peripheral T-cell lymphoma.

A 6o-year-old man was referred to Korea University Anam Hospital on day 130 post-HSCT because of dyspnea (modified Medical Research Council score 3) and a dry cough for 2 weeks. He was a 15pack per year ex-smoker. Two years earlier, he had been diagnosed with peripheral T-cell lymphoma and received six cycles of chemotherapy with cyclophosphamide, doxorubicin, vincristine, and prednisone (CHOP). As the disease progressed, he underwent a second chemotherapy protocol of etoposide, steroids, cytarabine, and cisplatin (E-SHAP). After four courses of E-SHAP, he was treated with autologous HSCT as a curative attempt. He denied any infection symptoms, such as yellowish sputum, fever, or chills. Physical examination revealed inspiratory crackles and expiratory wheezing in all lung fields. Routine laboratory analysis found the following: hemoglobin $11.7 \mathrm{~g} / \mathrm{dL}$, white blood cell count $8,700 / \mu \mathrm{L}$, and C-reactive protein $3.18 \mathrm{mg} / \mathrm{L}$. Blood chemistry revealed total protein $8.82 \mathrm{~g} / \mathrm{dL}$, albumin $4.5 \mathrm{~g} / \mathrm{dL}$, aspartate aminotransferase $144 \mathrm{IU} / \mathrm{L}$, alanine transaminase $291 \mathrm{IU} / \mathrm{L}$, and total bilirubin $1.16 \mathrm{mg} / \mathrm{dL}$, suggestive of hepatic injury of unknown etiology. Pulmonary function testing showed the restrictive pulmonary function defects of a markedly decreased total lung capacity, forced vital capacity (FVC), and forced expiratory volume in 1 second $\left(\mathrm{FEV}_{1}\right)$, but a normal ratio of $\mathrm{FEV}_{1}$ to FVC (FEV $/$ /FVC ratio) (Table 1). Chest radiography revealed cardiomegaly with a newly developed patchy opacity in the right upper lobe (RUL) (Fig. 1A). High-resolution computed tomography of the chest revealed non-calcified nodules with a branching pattern suggestive of bronchiolitis in the RUL and air trapping in the left lingular segment and right lower lobe (Fig. $1 \mathrm{~B}$ and $\mathrm{1C}$ ). To establish an exact diagnosis, a video-assisted thoracoscopic surgical biopsy of the posterior segment of the RUL was conducted. Histological examination showed an intraepithelial bronchiolar lymphocytic infiltration (Fig. 2A) and perivascular lymphocytic cuffing (Fig. 2B), highly suggestive of GVHD. An 

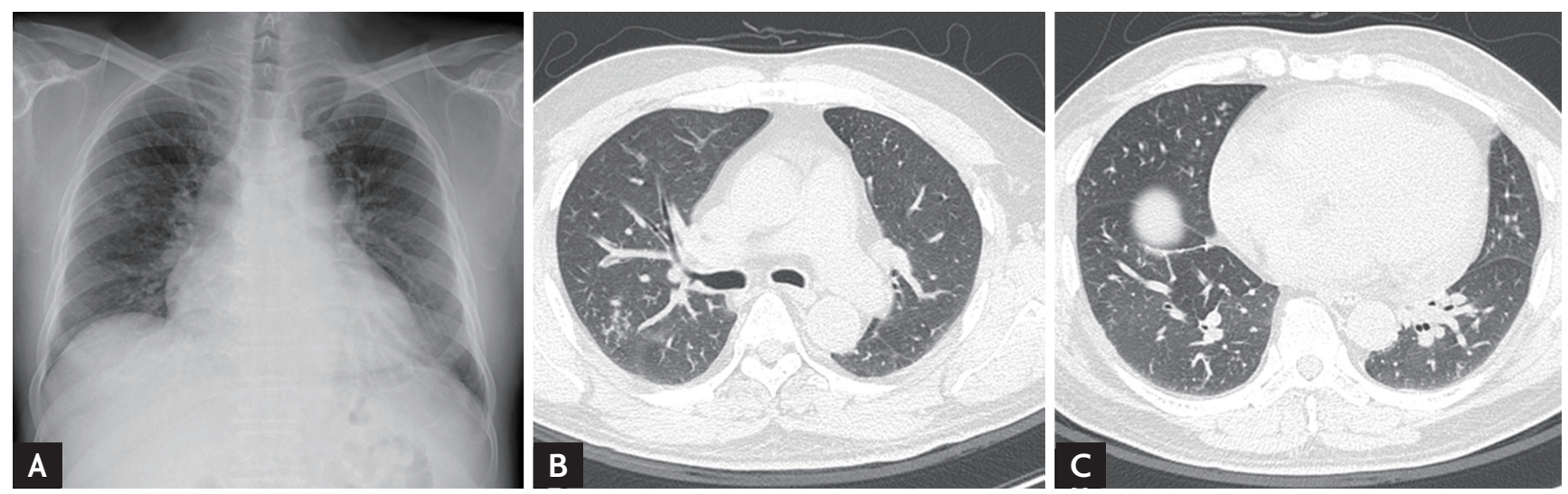

Figure 1. Chest posteroanterior radiography (A) and computer tomography (B, C) showed a newly developed patchy opacity suggestive of bronchiolitis in the right upper lobe. Air trapping and geographic hypoattenuation were also seen in the left lingular segment and right lower lobe.

inflammatory cellular infiltrate of mostly CD3-positive T lymphocytes was seen (data not shown). Histology revealed an underlying organizing pattern (Fig. 2C) of atypical cells mimicking viral cytoplasmic inclusions in airways and alveolar walls (Fig. 2D). However, immunohistochemical studies revealed no evidence of viral infection, such as herpes simplex viruses, cytomegalovirus, or Epstein-Barr virus. Consequently, the patient was diagnosed with pulmonary auto-GVHD.

Auto-GVHD is a self-limiting disease, and the patient was followed closely without additional management. His respiratory symptoms and lung function had improved slightly after 5 months (Table 1).

Auto-GVHD is usually milder than classical GVHD and is often self-limiting. It can occur spontaneously, or develop during immunomodulatory treatment using cyclosporine A (CsA) or interferon $\gamma$ after organ transplan-

Table 1. Initial and follow-up pulmonary function testing

\begin{tabular}{lcc}
\hline Variable & Initial & $\begin{array}{c}\text { 5-Month } \\
\text { follow-up }\end{array}$ \\
\hline FVC, L & $1.93(44)$ & $3.06(72)$ \\
$\mathrm{FEV}_{1}, \mathrm{~L}$ & $1.51(47)$ & $2.44(77)$ \\
$\mathrm{FEV}_{1} / \mathrm{FVC}, \%$ & 79 & 80 \\
$\mathrm{TLC}_{1}, \mathrm{~L}$ & $3.84(64)$ & $4.36(73)$ \\
Dlco, \% & 69 & 69 \\
\hline
\end{tabular}

Values are presented as number (\%).

FVC, forced vital capacity; $\mathrm{FEV}_{1}$, forced expiratory volume in 1 second; TLC, total lung capacity; Dlco, diffusing capacity for carbon monoxide. tation [2]. It may be caused by paradoxical self-reactive $\mathrm{CD} 8+\mathrm{T}$ cells recognizing a self-peptide in association with a major histocompatibility complex (MHC) class II molecule caused by CsA, or inhibition of peripheral tolerance by depletion of regulatory $\mathrm{T}$ cells following preparative chemotherapy [2]. One study reported non-infectious pulmonary complications on chest computed

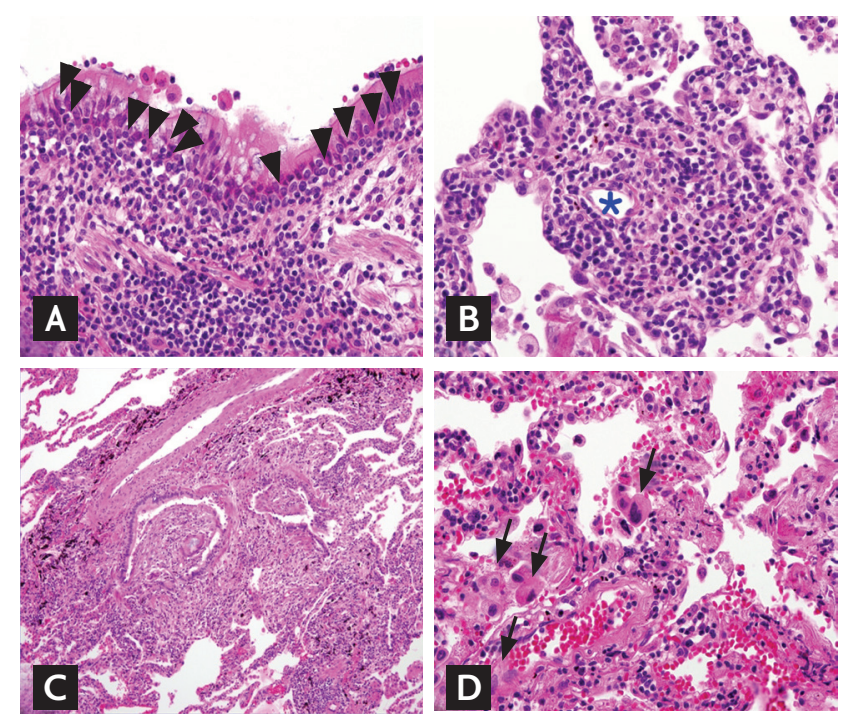

Figure 2. Video-assisted thoracoscopic surgical lung biopsy revealed intraepithelial bronchiolar T lymphocytic infiltrations (arrowheads; A, H\&E, ×200) and perivascular lymphocytic cuffing (asterisk; B, H\&E, ×200) highly suggestive of graft-versus-host disease. (C) An underlying organizational pattern was also noted (H\&E, ×40). (D) Atypical cells mimicking viral cytoplasmic inclusions (arrows) in the airways and alveolar walls were present without evidence of viral infection $(\mathrm{H} \& \mathrm{E}, \times 2 \mathrm{OO})$. 
tomography in patients with pathologically diagnosed GVHD after allogeneic HSCT [3]. The most common finding was geographic hypoattenuation suggestive of bronchiolitis obliterans, which is a recognized pulmonary manifestation of chronic GVHD. Other findings included ground-glass attenuation, reticulation, air trapping, and a "crazy paving" pattern. Histopathologically, pulmonary GVHD is characterized by intrabronchiolar $\mathrm{CD}$ 3-positive $\mathrm{T}$ cell infiltration, apoptosis, and perivenulitis, findings that help to discriminate GVHD from infections [4]. In this study, pulmonary GVHD was classified into acute lung injury, organizing pneumonia, and chronic interstitial pneumonia patterns.

In conclusion, we report a rare case of biopsy-proven pulmonary auto-GVHD. Although pulmonary auto-GVHD has good outcomes similar to auto-GVHD in other organs, it may have a broad spectrum of clinical, radiological, and pathological features. To our knowledge, this is the first case of pulmonary auto-GVHD reported in Korea.

Keywords: Lung; Autologous graft-versus-host disease; Hematopoietic stem cell transplantation

\section{Conflict of interest}

No potential conflict of interest relevant to this article was reported.

\section{REFERENCES}

1. Hood AF, Vogelsang GB, Black LP, Farmer ER, Santos GW. Acute graft-vs-host disease: development following autologous and syngeneic bone marrow transplantation. Arch Dermatol 1987;123:745-750.

2. Kline J, Subbiah S, Lazarus HM, van Besien K. Autologous graft-versus-host disease: harnessing anti-tumor immunity through impaired self-tolerance. Bone Marrow Transplant 2008;41:505-513.

3. Song I, Yi CA, Han J, et al. CT findings of late-onset noninfectious pulmonary complications in patients with pathologically proven graft-versus-host disease after allogeneic stem cell transplant. AJR Am J Roentgenol 2012;199:581-587.

4. Xu L, Drachenberg C, Tavora F, Burke A. Histologic findings in lung biopsies in patients with suspected graftversus-host disease. Hum Pathol 2013;44:1233-1240. 